\title{
Fabrication of nanometer size photoresist wire patterns with a silver nanocrystal shadowmask
}

\author{
S. H. Choia) and K. L. Wang \\ Electrical Engineering Department, University of California at Los Angeles, Los Angeles, \\ California 90095-1594 \\ M. S. Leung, G. W. Stupian, and N. Presser \\ Electronics Technology Center, The Aerospace Corporation, El Segundo, California 90245-4691 \\ S. W. Chung, G. Markovich, S. H. Kim, and J. R. Heath \\ Department of Chemistry and Biochemistry, University of California at Los Angeles, Los Angeles, \\ California 90095-1569
}

(Received 13 October 1998; accepted 19 April 1999)

\begin{abstract}
In this article, we present a new method for fabricating precisely defined nanometer scale photoresist wire patterns. The Langmuir technique was utilized to form high aspect ratio lamellae, or wire patterns, of Ag nanocrystals at the air/water interface, and these patterns were transferred onto resist-coated substrates as a Langmuir-Schaeffer film and as a shadowmask. The wire patterns were transferred to the photoresist material by spatially selective electron beam exposure on the $\mathrm{Ag}$ nanocrystal wire shadowmask. Monte Carlo simulation was done to estimate the electron stopping power for the Ag nanocrystal shadowmask at low voltage. (C) 1999 American Vacuum Society. [S0734-2101(99)20104-2]
\end{abstract}

\section{INTRODUCTION}

There is much interest in using low dimensional semiconductor nanostructures for future electronic, optoelectronic, and magnetoelectronic device applications. ${ }^{1,2}$ To fabricate semiconductor structures in the nanometer range, it is necessary to develop lithographic techniques with nanometer-scale resolution. Much effort has been made in fabricating nanometer-scale structures using high-energy, highly defined sources such as electron beams and $\mathrm{x}$ rays. ${ }^{3}$ The proximity effect, caused by the high-energy sources, limits the resolution of lithography. Scanning techniques with a low energy source, such as scanning tunneling microscope lithography, are not suitable for high throughput. ${ }^{4,5}$ One possibility is to utilize self-assembly techniques to produce shadowmasks that are characterized by periodic, nanometer-scale patterns. Block copolymers, for example, will phase separate into structures with nanometer-scale periodicities. ${ }^{6}$ However, these organic materials are poor contrast shadow masks. Another option is to use higher-contrast inorganic systems. Organically functionalized metal and semiconductor nanocrystals $(2-100 \mathrm{~nm})^{7,8}$ can assemble into a variety of organized structures, ${ }^{9}$ including lamellar (wire-like) phases. ${ }^{10}$ These structures usually consist of self-oriented high-aspect ratio nanocrystals, and can be transferred as Langmuir-Schaeffer (horizontal lift-off) films to virtually any type and size of substrate. In this article, we describe the use of structured nanocrystal phases as resist masks and the subsequent transfer of the patterns. We demonstrate that the combined use of low energy electron beam exposure and self-assembled nanocrystal shadowmasks, may be a good candidate as a low-cost fabrication technique for semiconductor nanometerscale structures.

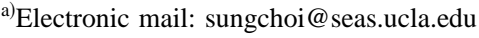

\section{EXPERIMENTAL RESULTS}

We utilized a method ${ }^{11}$ to prepare size-selected, 3-nmdiam octanethiol-capped $\mathrm{Ag}$ nanocrystals in a $1-2 \mathrm{mg} / \mathrm{ml}$ hexane solution. Langmuir films were formed on a Nima Technology type 611 Langmuir trough at $15^{\circ} \mathrm{C}$. In most experiments, only one drop $(\sim 3 \mu l)$ of the $\mathrm{Ag}$ nanocrystal solution was dispersed on the water surface. The nanocrystals spontaneously self-assembled into high-aspect ratio wire-like structures. ${ }^{10}$ The physics that lead to this assembly process involve competing interactions among the nanocrystals on the water surface, and will be described elsewhere. ${ }^{12}$ The width of the wires could be controlled from 20 to 300 $\mathrm{nm}$, and for a given set of wires, a narrow distribution of widths $(15 \%-25 \%)$ could be obtained. The interwire distance, as well as the alignment of the wires, could be controlled via compression of the wires on the Langmuir trough. The wires were found to keep their structures and aligned with interwire separations of just a few nanometers. The nanocrystal Langmuir film was then transferred as a Langmuir-Schaeffer film to polymethyl methacrylate (PMMA) coated substrates. This nanocrystal pattern was amplified by immersing the substrate in a solution containing decanedithiol $\left(\mathrm{HSC}_{10} \mathrm{H}_{20} \mathrm{SH}\right)$ followed by immersing the substrate into a hexane/nanoparticle solution. This amplification slightly increased the width of the wires, and it doubled the height of the wires to about $8 \mathrm{~nm}$. All substrates used were prepared within a couple of days. Figure 1 shows a field emission scanning electron microscopy (FESEM) micrograph of the transferred Ag nanocrystal films. The nanocrystal film was transferred at a surface pressure of 15-20 $\mathrm{mN} / \mathrm{m}$, showing a wire-like structure with a very narrow distribution of wire widths for a given sample. A certain number of the wires as long as 1-2 $\mu \mathrm{m}$ length were found to align in a regular pattern. 


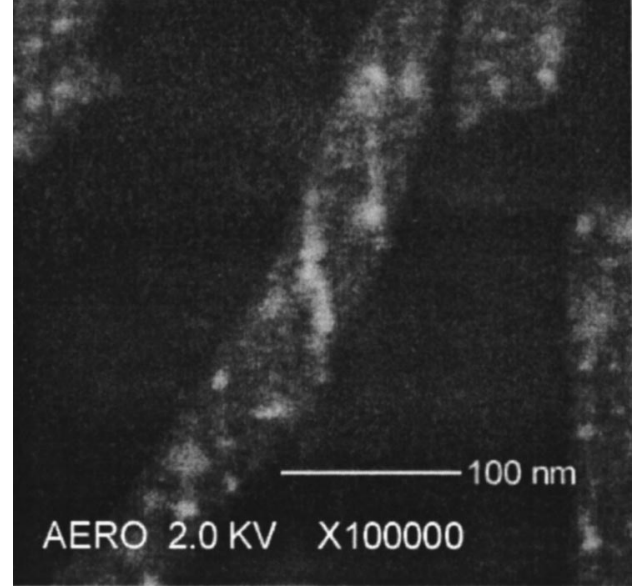

FIG. 1. FESEM micrograph of a silver nanocrystal wire.

Low energy electron beam exposure was used since low energy electrons have less lateral scattering, higher interaction cross sections, and shorter substrate penetration depths than the higher-energy electrons used in conventional electron beam lithography. To estimate the electron stopping power $(R)$ for silver nanocrystals, the formula for the electron range given by Kanaya and Okayama was used ${ }^{13}$

$$
\left.\rho R=\frac{0.0276 A E^{1.67}}{Z^{0.889}} \quad \text { (in micrometers }\right),
$$

where $E$ is the acceleration voltage in kilovolts, $Z$ the atomic number, and $\rho$ the density in grams per cubic centimeter. The estimated electron stopping effective range $(R)$ for silver at $700 \mathrm{~V}$ is $4 \mathrm{~nm}$, which is less than the thickness of silver nanocrystal wires. A Monte Carlo approach with more than $10^{5}$ electron trajectories through the silver was used to evaluate the electron stopping power of the Ag nanocrystal shadowmask. As shown in Fig. 2, the penetration depth was found to be about $4 \mathrm{~nm}$ at $700 \mathrm{~V}$, in agreement with the value from Eq. (1). This penetration depth is bigger than the diameter of Ag nanocrystals but is smaller than the thickness of the amplified nanocrystal wires. A JEOL 6401F FESEM was used to expose the sample to a $600 \mathrm{pA}, 700 \mathrm{~V}$ electron beam with the field size of $100 \mu \mathrm{m} \times 100 \mu \mathrm{m}$ for $10 \mathrm{~min}$ to expose the PMMA with an electron dose of $50 \mu \mathrm{C} / \mathrm{cm}^{2}$. At $700 \mathrm{~V}$, only very thin resist films $(\leqslant \sim 50 \mathrm{~nm})$ can be exposed. The entire process of pattern transfer and development is illustrated in Fig. 3. Ag nanocrystal wires of $4 \mathrm{~nm}$
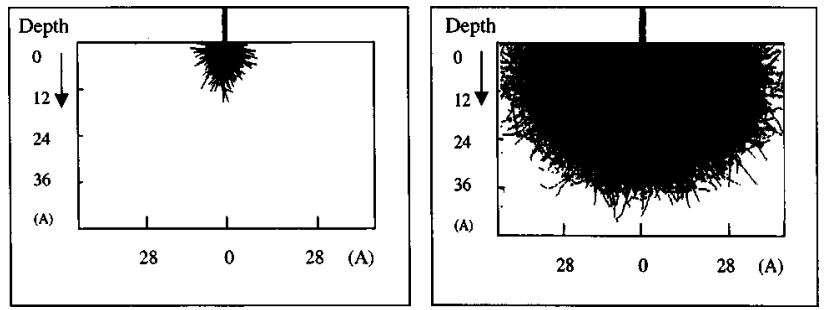

FIG. 2. Schematic of the electron stopping effectiveness at $500 \mathrm{~V}$ (left) and $700 \mathrm{~V}$ (right) for silver from a Monte Carlo simulation.

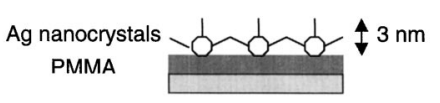

(a)

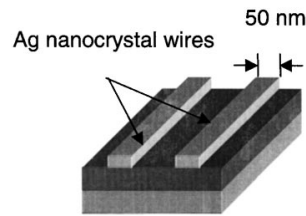

(c)

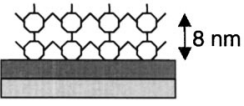

(b)

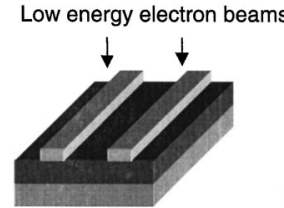

(d)

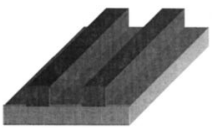

(e)
FIG. 3. Procedure of low energy electron beam lithography with a silver nanocrystal shadowmask. (a) Deposition of silver nanocrystal wires on the PMMA. (b) Formation of metallized and amplified silver nanocrystal wires. The distance between the wires is reduced due to the metallization process. (c) Transferring of silver nanocrystal wires onto the PMMA. (d) Low energy electron beams onto silver wires/PMMA. (e) Formation of PMMA wire patterns after developing.

thickness $50 \mathrm{~nm}$ width were transferred on the PMMA coated substrates by the Langmuir-Blodgett trough lift-off process as illustrated in Fig. 3(a). For better pattern transfer a thicker shadowmask is preferred. To accomplish this, amplification of the layer thickness was performed. Because the top of the Ag nanocrystal wires was hydrophobic, a second monolayer of $\mathrm{Ag}$ nanocrystal wires could be added to the previously deposited monolayer by the second LangmuirBlodgett trough lift-off process as illustrated in Fig. 3(b). The amplified Ag nanocrystal structures were kept in air for a few days to allow for organic ligands to evaporate. The occupied areas by organic ligands between the nanocrystals in the wire decreased through a metallization process in air. As a result of the amplification and metallization process, 10-nm-high Ag nanocrystal wires were formed and the interwire distance was reduced as illustrated in Figs. 3(b) and 3(c). The amplified and metallized Ag wires on the PMMA were then exposed to a low energy electron beam as shown in Fig. 3(d). Following the e-beam exposure, the patterns were developed for $1 \mathrm{~min}$ in a mixture of methyl isobutyl ketone and isopropanol with a ratio 1:3. Figure 4 shows the

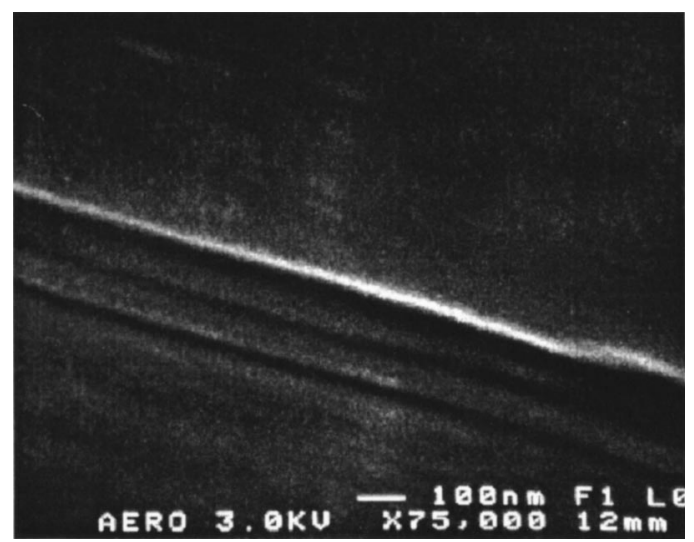

FIG. 4. FESEM micrographs of the 50-nm-wide PMMA wire patterns by a silver nanocrystal shadowmask after developing process. 


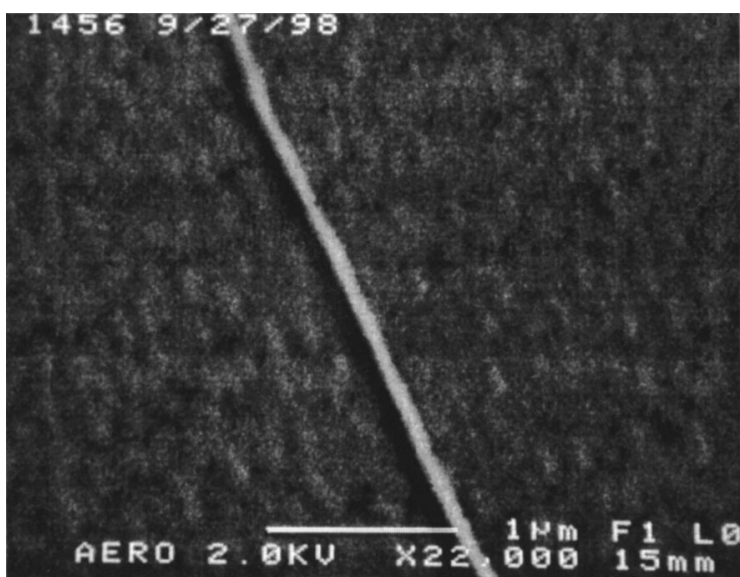

FIG. 5. FESEM micrograph of the 50-nm-wide silicon wire by a subsequent RIE process.

FESEM micrograph of the 50-nm-wide, 10-nm-high PMMA wire patterns fabricated by a Ag nanocrystal shadowmask after developing process. A subsequent anisotropic reactive ion etching (RIE) process, with a $\mathrm{CF}_{4}$ to $\mathrm{O}_{2}$ mixture of 22-18 mTorr, and the plasma sustaining power of $150 \mathrm{~W}$, resulted in about 50-nm-wide silicon wire structures as shown in Fig. 5.

\section{CONCLUSIONS}

In conclusion, a parallel array of 50-nm-wide PMMA wire patterns was fabricated by low energy electron beam exposure using Ag nanocrystals as a shadowmask. The photoresist wire patterns obtained using the nanocrystal shadowmask were used to transfer the pattern to silicon substrates by a subsequent RIE process. The major technological advantage of the present technique in preparing quantum wires is that it allows for the use of low energy electron beam exposure $(700 \mathrm{~V})$, which reduces the proximity effects. This technique is suitable for low-cost and high throughput fabrication of quantum wires.

\section{ACKNOWLEDGMENTS}

This work was partially supported by the Augmentation Awards for Science \& Engineering Research Training (AASERT) Fellowship Grant No. N00014-96-1-1258, the Office of Naval Research MURI, and ARO MURI and the U.S. Air Force under Contract No. F04701-93-C-0094. One of the authors (J.R.H.) acknowledges the Office of Naval Research Contract No. N00014-981-0422, and the Packard Foundation. The authors also wish to thank M. Tuelling and R. Robertson at the Aerospace corporation for useful assistance.

${ }^{1}$ A. P. Alivisatos, Science 271, 933 (1996).

${ }^{2}$ L. F. Thompson, C. G. Willson, and M. J. Bowden, Introduction to Microlithography, ACS Symp. Series 219 (American Chemical Society, Washington DC, 1983).

${ }^{3}$ M. A. McCord and R. F. W. Pease, J. Vac. Sci. Technol. B 4, 86 (1996). ${ }^{4}$ P. I. Majundar, J. P. Oden, L. A. Carrejo, J. I. Nagahara, and J. Alexander, Appl. Phys. Lett. 61, 2293 (1992).

${ }^{5}$ T. H. P. Chang, D. P. Kern, E. Kratschmer, K. Y. Lee, H. E. Luhn, M. A. McCord, S. A. Rishton, and Y. Vladimirsky, IBM J. Res. Dev. 32, 462 (1988).

${ }^{6}$ R. W. Zehner, W. A. Lopes, T. L. Morkved, H. Jaeger, and L. R. Sita, Langmuir 14, 242 (1998).

${ }^{7}$ C. B. Murray, D. J. Norris, and M. G. Bawendi, J. Am. Chem. Soc. 115, 8706 (1993).

${ }^{8}$ M. Brust, M. Walker, D. Bethell, D. J. Schiffrin, and R. Whyman, J. Chem. Soc. Chem. Comm. 801 (1994).

${ }^{9}$ C. B. Murray, C. R. Kagan, and M. G. Bawendi, Science 270, 1335 (1995).

${ }^{10}$ S. W. Chung, G. Markovich, and J. R. Heath, J. Phys. Chem. B 102, 6685 (1998).

${ }^{11}$ J. R. Heath, C. M. Knobler, and D. V. Leff, J. Phys. Chem. B 101, 189 (1997).

${ }^{12}$ R. P. Sear, S. W. Chung, G. Markovich, W. M. Gelbart, and J. R. Heath (submitted).

${ }^{13}$ J. I. Godstein, D. E. Newbury, P. Echlin, D. C. Joy, C. Fiori, and E. Lifshin, Scanning Electron Microscopy and X-Ray Microanalysis (Plenum, New York, 1981), p. 83. 